\title{
Using Remote Telescopes for Exoplanet Searches
}

Richard P. Olenick ${ }^{1 *}$, Arthur Sweeney ${ }^{1}$, Laura Aumen ${ }^{1,2}$, Ramses Gonzalez ${ }^{1,3}$, Alex Henderson ${ }^{1,3}$, Mark Rodriguez ${ }^{1}$, Philip Lenzen ${ }^{1}$, John Paul Jones ${ }^{1}$

\begin{abstract}
The Small Telescope Extrasolar Transit Search (STExTS) project involves undergraduates in research using ground-based small aperture, wide-angle telescopes to search for hot Jupitersize transiting exoplanets of stars down to 13th magnitude. The observational campaigns in 2015 and 2016 used the Monroe Observatory of the University of North Texas with a f/1.5 152 $\mathrm{mm}$ astrograph installed for remote observing and in 2017 twin f/1.25 $152 \mathrm{~mm}$ astrographs were remotely accessed at the Dark Sky Observatory Collective (DSOC) near Ft. Davis, TX. Hardware and the use of commercial software for remote operation of the telescope and camera were installed and coordinated by the team. Observational campaigns usually run 25 to 35 nights, capturing 5000+ stars per image, 250 images per night of the same region of the sky. A software processing pipeline and SQL database were created for the searches. The pipeline examines the images, calibrates them, extracts the stars, and matches each star with an astronomical catalog of stars for identification. Finally photometric analysis is performed to measure the light curve of every star, the results of which are stored in the project SQL database hosted by the University of Dallas. PERANSO and VARTOOLs are used to analyze the light curve and to identify stars of interest. The process of converting the astrograph to remote use, the development of the data pipeline, the role of student researchers, and a new exoplanet candidate, GSC 2087-1126 b, are presented.
\end{abstract}

\section{Keywords}

techniques: photometric; methods: data analysis; planets and satellites: detection

${ }^{1}$ Department of Physics, University of Dallas, Irving TX USA

${ }^{2}$ Department of Physics, Creighton University, Omaha NE USA

${ }^{3}$ University of North Carolina, Chapel Hill NC USA

*Corresponding author: olenick@udallas.edu

\section{Introduction}

Exoplanets with large magnitude depths often transit bright host stars, allowing ground-based, photometric measurements of flux over time to be acquired with differential photometry on even modest astronomical equipment. Since the first transiting exoplanet discovery by Charbonneau (Charbonneau et al., 1999) in 1999 several ground based small telescope search projects have succeeded in finding numerous exoplanets, most notably WASP (Cameron et al., 2007) and KELT (Pepper et al., 2007), through the transit method.
The transit depth scales as

$$
\left(\frac{\Delta F}{F}\right) \approx 0.01\left(\frac{r}{R_{J}}\right)^{2}\left(\frac{R}{R_{S u n}}\right)^{-2}
$$

where $r$ and $R$ are the planet and star radii, respectively. For Jupiter transiting the Sun, taking Jupiter's radius as $R_{J} \approx 0.1 R_{S u n}$, the transit depth is $\sim 1 \%$. For an edge-on orbit, the transit time is given by 
$\Delta t=\frac{P R}{\pi a}=3\left(\frac{P}{4}\right)^{1 / 3}\left(\frac{R}{R_{\text {Sun }}}\right)\left(\frac{M}{M_{\text {Sun }}}\right)^{-1 / 3}$

where $M$ is the star's mass and $P$ is the orbital period in days, and $a$ is the semimajor axis of the planet about the star. Therefore, for hot Jupiters transiting sun-like stars, a $1 \%$ decrease in flux and a transit length of 3 hours is expected and is easy to detect with modern CCD cameras. Since transits occur only when the orbit is close to edge-on, that is, for orbital inclinations $i<90^{\circ}$, the transits become shallower and narrower and vanish when $\cos (i)<(R+r) / a$. Consequently, for random orientations of orbits, the probability of seeing a transit is (Haswell, 2010)

Prob $=\frac{R+r}{a} \approx 0.1\left(\frac{P}{4}\right)^{-2 / 3}\left(\frac{R}{R_{\text {Sun }}}\right)\left(\frac{M}{M_{\text {Sun }}}\right)^{-1 / 3}$

For a hot Jupiter, $P \approx 4 \mathrm{~d}, a \approx 0.05$ AU, so the transit probability is $\sim 10 \%$. By comparison, for an Earth-size planet ( $P=1 \mathrm{yr}, a=1 \mathrm{AU})$, the transit probability is only $0.5 \%$.

The Small Telescope Extrasolar Transit Search (STExTS) project is a ground-based small aperture, wide-angle telescope project involving undergraduates in research using remote observatories to search for transiting exoplanets of stars down to 13th magnitude. Section 2 describes the remote observatories, astrographs, and software used for operation. Section 3 describes the nightly routine used by undergraduates, the software processing pipeline and SQL database that were created for the searches. Section 4 describes the methods and software used in analyses. The STExTS team has found candidates for exoplanets, as well as discovering numerous new RR Lyrae variable stars (Melendez et al., 2014) and W UMa binaries (Schneiderjan et al. 2013, Meier et al. 2013). In Section 5 we present the results of observations and analysis of a new exoplanet candidate, GSC 2087-1126 b, and discuss its physical parameters. In Section 6 we summarize the methods and results of the project.

\section{Experimental Design}

The Small Telescope Exoplanet Transit Search (STExTS) project at the University of Dallas has been in operation since 2012. For approximately 35 nights each summer, the team observes the same single patch of sky through wide-angle astrographs, in order to gather data on a large number of stars over an extended period. Time-resolved CCD photometry was conducted with a f/1.5 $152 \mathrm{~mm}$ astrograph with a $3^{\circ}$ field of view and a f/1.5 152 $\mathrm{mm}$ astrograph with a $0.8^{\circ} \mathrm{FOV}, \mathrm{SBIG}$ ST- $10 \mathrm{CCD}$ cameras, and a Paramount MEII mount. The telescope specifications are summarized in Table 1. Target regions were in Hercules, Vulpecula, and Cygnus - all outside of WASP and Kepler target areas.

Table 1. Telescope Specifications

\begin{tabular}{cccccc}
\hline & $\begin{array}{c}\text { Objective } \\
\text { Diameter (mm) }\end{array}$ & $\begin{array}{c}\text { Focal } \\
\text { Length (mm) }\end{array}$ & $\begin{array}{c}\text { Stopped } \\
\text { down to }\end{array}$ & $\begin{array}{c}\text { Image } \\
\text { Scale ("/pix) }\end{array}$ & $\begin{array}{c}\text { Image } \\
\text { Size (min) }\end{array}$ \\
\hline Fat Boy & 152 & 230 & $\mathrm{f} / 2.8$ & 6.14 & $151 \times 224$ \\
Serena & 152 & 730 & $\mathrm{f} / 4.8$ & 1.92 & 47 X 70 \\
\hline
\end{tabular}

In 2012 - 2014 the STExTS telescopes were set up in an area with very low light pollution at a 9200 foot $(2800 \mathrm{~m})$ elevation in Pitkin, Colorado. In the 2014, observations were done on the shore of a small lake in the Ottawa National Forest in the Upper Peninsula, Michigan. Since 2015 the project has configured the telescopes for remote observing at the University of North Texas Monroe Observatory outside Gainesville, TX and the Dark Sky Observatory Collaborative (DSOC) near Ft. Davis, TX.

Two scopes were originally used to collect data that were christened "Fat Boy" and "Serena" by students to differentiate them. Fatboy is a 6-in (152 $\mathrm{mm}$ ) astrograph constructed in-house from a professional-grade television camera lens and Serena is a 6-in (152 mm) astrograph constructed from components. In 2017 the project moved to using two identical Serena-type telescopes, Castor and Pollux, that were constructed in house. These two telescopes are mounted slightly off axis to each other on a platform on the MEII mount, as 


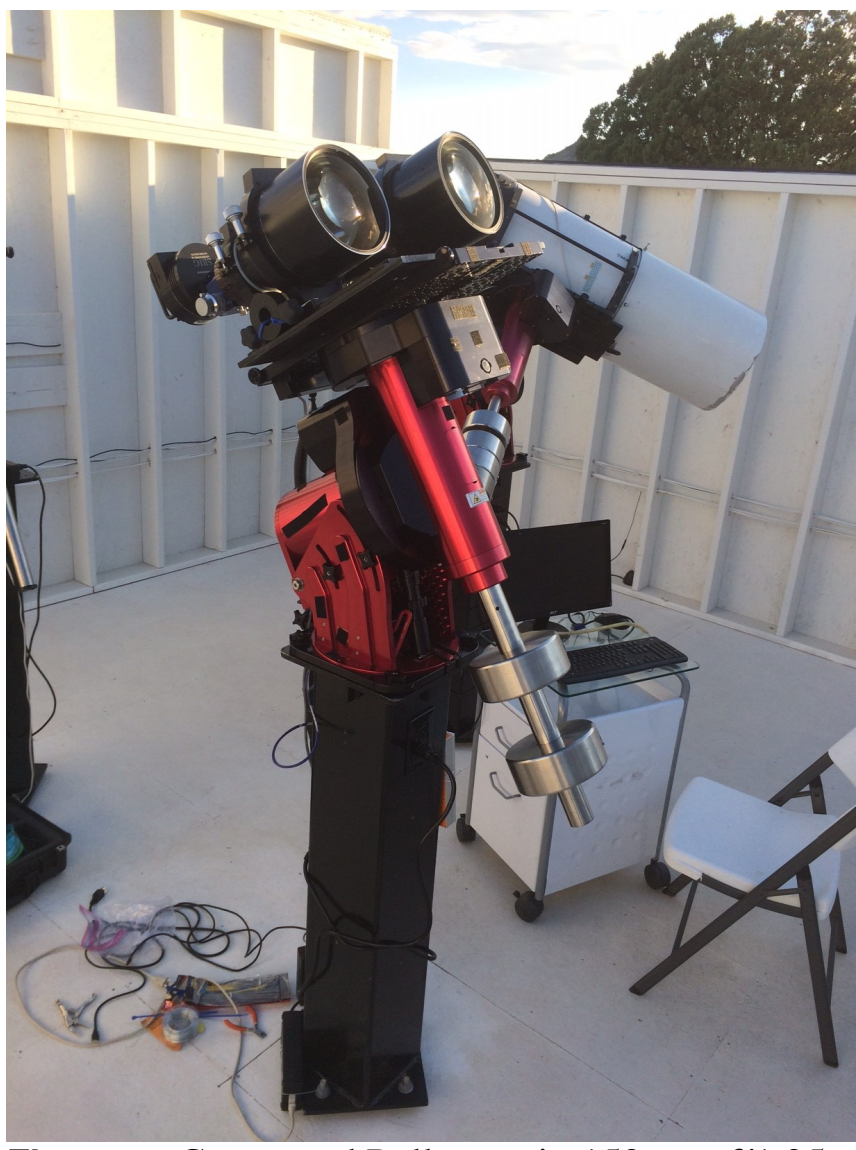

Figure 1. Castor and Pollux, twin $152 \mathrm{~mm} \mathrm{f/1.25}$ astrographs on a Paramount MEII mount are shown at the Dark Sky Observatory Collaborative near Ft. Davis, TX.

seen in Fig.1. Johnson R-band filters are used on all telescopes.

Undergraduate student researchers learn how to control the telescopes and to take data remotely. The students take data for two months, weather permitting, from their residences and reduce images into photometric data. They also use various software packages for analyses (PERANSO, VARTOOLS, etc.). Students are also required to write a 35-page thesis on their work with portions ready for incorporation in publications.

\section{Observations}

Typical observation sessions last six hours during which images are taken with a 60 -second integration time. The images are centered on a

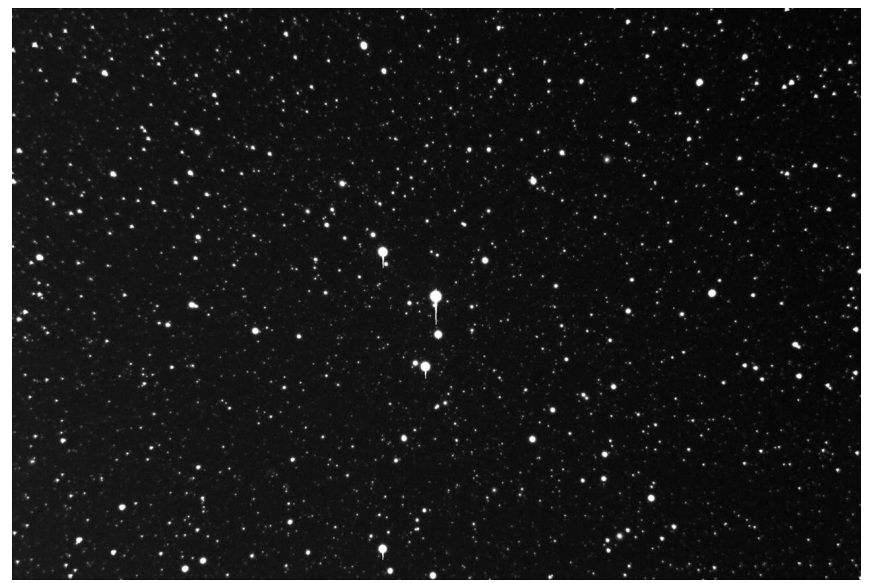

Figure 2. The three-degree field of view of the astrograph Fatboy centered on SAO 85344. The blooming of the central star can be seen; however no data was taken on that star. As described in the text, approximately 5000 stars can be extracted from each image.

bright star for each campaign, which is used for image identification. Figure 2 shows the field of view from our 2014 campaign in which SAO 85344 was used for identification. Tracking is accomplished with the internal CCD guide chip in the ST-10 camera. Sixteen bias and sixteen dark frames for calibration are collected each night. Sixteen flat fields are taken on alternate nights. Dark and bias frames are taken at same temperature as data frames. Raw images, flats, bias, and darks are acquired with Maxim DL. All image frames are calibrated using AIP4Win (Berry and Burnell, 2000), utilizing dark frames for removing CCD thermal noise, bias frames for removing digitizing and readout noise, and flat fields for removing CCD pixel sensitivity variances and to correct for vignetting. Figure 3 shows the nightly routine followed by the undergraduate researchers. Access to the remote observatory is achieved with Teamviewer and The SkyX is used to interface with the telescopes.

\section{Data Pipeline}

Each image is passed to PinPoint astrometric engine (Denny, 2006) software for photometric and astrometric processing. Given approximate coordinates from the FITS header file and input 


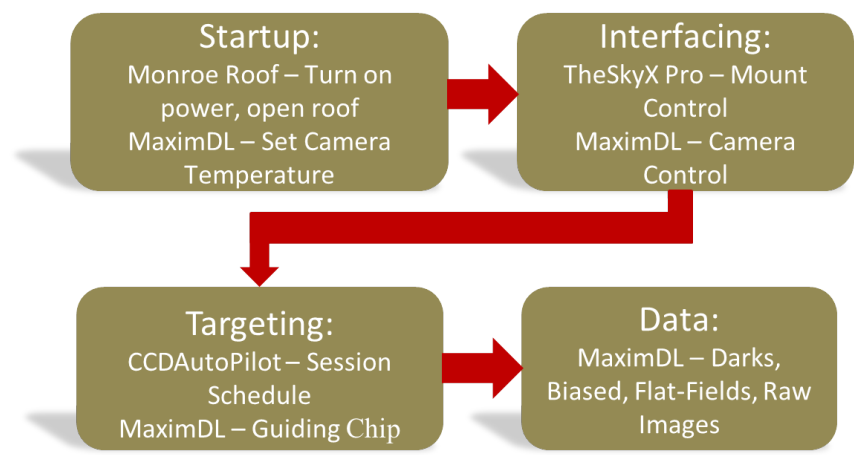

Figure 3. The nightly routine followed by undergraduates taking data indicating the software components utilized by them.

from the GSC 1.1 star catalog, PinPoint obtains an astrometric solution for the plate (image). PinPoint is configured to load subsequently Source

Extractor (SExtractor) (Bertin and Arnouts, 1996). SExtractor is a program that builds a catalog of objects from an astronomical image using the USNO-B1.0 catalog. Although it is particularly oriented towards reduction of large scale galaxy-survey data, with thresholds and nets adjusted, it performs reasonably well on moderately crowded star fields. SExtractor extracts the $(x, y)$ position of each star and passes this, along with its measured magnitude, back to PinPoint. Given the right ascension and declination, a local catalog is generated of the field area from the USNO-B1.0 catalog that is accessed to identify each star. The locally generated catalog is necessary because PinPoint does not provide the name of all of extracted stars. We use the VizieR Catalog Service to download and create a subset catalog based on the centers of our plates and their field of view of about 3 degrees. A raw data SQL file is created that contains data on each processed image and a raw data file that contains data on each star identified. If a star cannot be identified, it is given a unique name based on its coordinates. The software pipeline does not determine a standard precise magnitude. The determined magnitude is only an approximate magnitude based on the $\mathrm{R}$-band reported magnitudes contained in the USNO-B1.0 catalog that is used in our analysis. We are interested mainly in the differential changes in a star's light curve throughout the observation periods.

For each star in the raw data file, each night of data is processed. Each star in each night of data is analyzed for its number of observations and consistency of magnitudes. A modified Z score is calculated, based on the median absolute deviation (MAD), for each data point during the night. If the $\mathrm{Z}$ score is greater than 3.5 the data point is considered an outlier and discarded. If, during a night of observation, a given star is missing for more than $25 \%$ of the images, all data points for the star, for that night, are flagged as suspect. Finally, each data point on each image for each night is compared to its spatially nearest four neighbors and if it deviates by more than $2 \sigma$ it is flagged in the database.

The final step in the main processing pipeline is performed by another custom program written that performs systematic error reduction using the SYSREM algorithm (Tamuz et al., 2005). The new calculated magnitudes are placed in another field of the SQL database.

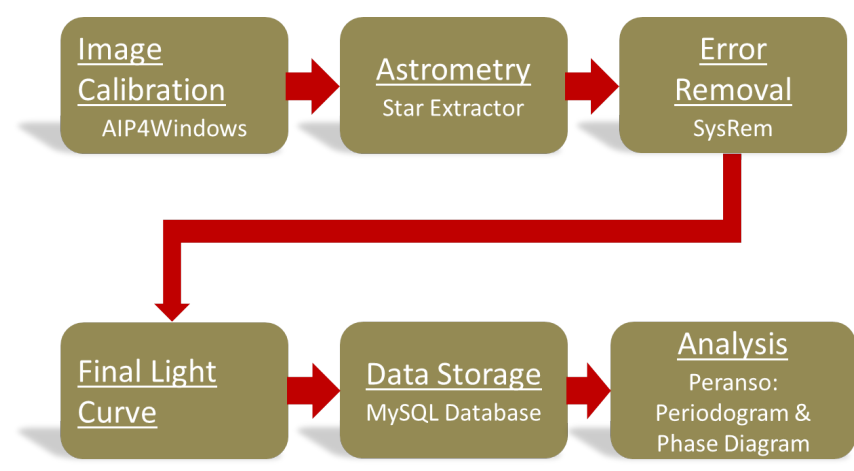

Figure 4. The flowchart shows the steps taken from observations to data storage and transit search.

\section{Observational Errors}

The presence of systematic (red) noise limits the detection efficiency of searches for transiting exoplanets (Pont et al., 2006). Consequently our surveys were limited by systematic noise and most significantly by random or stochastic noise in the data. We find that because we are interested 
primarily in the shape of a light curve and are implementing differential photometry, systematic noise is of little consequence because it is, in general, applicable to all stars and images.

The greatest source of noise in the STExTS project is stochastic noise, and in particular, Poisson noise, aperture pixel noise, scintillation noise and seeing noise. We believe that of these, seeing noise is by far the most significant.

A detailed measurement and analysis of a typical single image of our data indicates that, for example, the star GSC 2087- $0364(\mathrm{mag}=10.88)$ has a SNR of 171 and a standard error of $0.006 \mathrm{mag}$. This value comports with a theoretical calculation based on equipment used. We find the measured noise of the entire dataset for the star is $0.012 \mathrm{mag}$ for all images from a single night. We attribute this difference in error to sky conditions, mainly high clouds. We find, however, the measured noise of the entire dataset for the star is $0.026 \mathrm{mag}$. The preceding error analysis is performed before a SYSREM (systematic error removal) algorithm is applied and we find that the SYSREM algorithm reduces standard error $\sim 20 \%$ in certain cases/campaigns but does not completely remove the red and white (pink) noise.

Our astrometric analysis error as reported by PinPoint ranges from 0.5 to 1.5 arc-secs. The astrometric error has proven to not be a problem in pursuit of the overall project goals.

\section{Data Analysis}

The light curves of all stars in the SQL database are initially visually examined by undergraduate students to see if there is any indication of a periodic trend or transit-like decreases in brightness (Aumen et al., 2015). Often, one must zoom in on a single night's data in order get a good idea of whether there is a trend in the light curve since the typical detectable transit lasts only a few hours. A visual scan of a single light curve in PERANSO (Vanmunster, 2007) only takes a few seconds, but there are thousands of stars to analyze, even if we limit our analysis to stars between

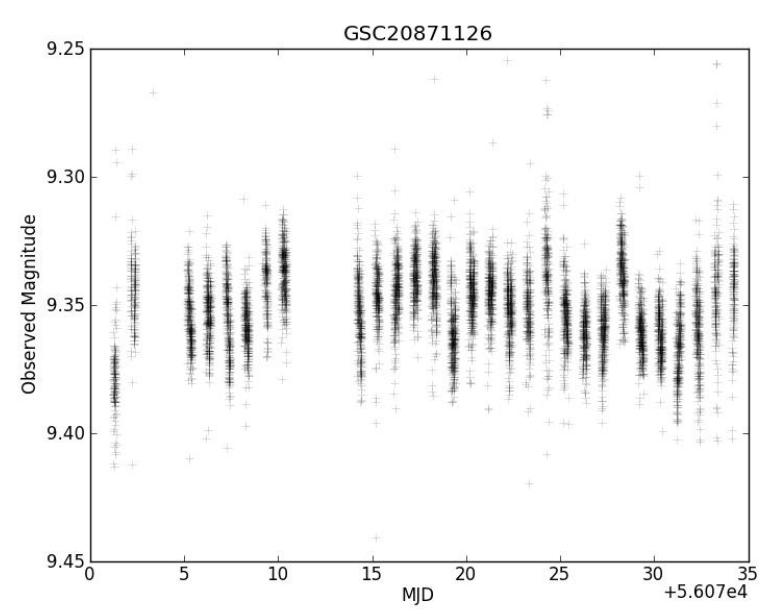

Figure 5. Observational light curve of GSC 2087-1126 for 35 nights of observations. The vertical axis gives the Johnson R-band magnitude and the horizontal axis the modified Julian date.

magnitude 9 and 13. Consequently, students spend much time on this non-automated aspect of the data analysis. Once a star of interest is identified, students perform differential photometry on the star of interest by using nearby stars in the database with constant magnitudes as check and comparison stars.

Periodograms are calculated for detrended data for the candidates of interest using the Lomb-Scargle method in PERANSO. The Lomb-Scargle periodogram (Lomb 1976, Scargle 1982) decomposes the data into a series of sine and cosine functions and is similar to least-squares 'statistical' methods, which minimize the differences between observed and modeled data. In PERANSO the Lomb-Scargle algorithm is optimized using the Horne and Baliunas method (Horne and Baliunas, 1986), which scales the periodogram by the total variance of the data, yielding a better estimation of the frequency of the periodic signal. The experimental approach taken by the project in taking 35 days of data of the same region lends itself to identifying transits by the folded light curves of the data in which several transits, whole or partial, may have been observed.

For the light curves of interest that might contain transits we use an EEBLS (Edge Enhanced 
Box-fitting Least Squares) algorithm (Heuser, 2013) in PERANSO to determine the period and produce a phase diagram. The box-fitting least squares (BLS) algorithm finds periodic alternation between levels. EEBLS augments the BLS by accounting for edge effects during transits. PERANSO displays the fit of the EEBLS method and calculates the epoch of mid-transit events, the transit depth, and duration. Figure 6 shows the phase diagram for GSC 2087-1126 when the BLS algorithm in PERANSO found a possible transit with a 0.79380 day period, which is shown in red.

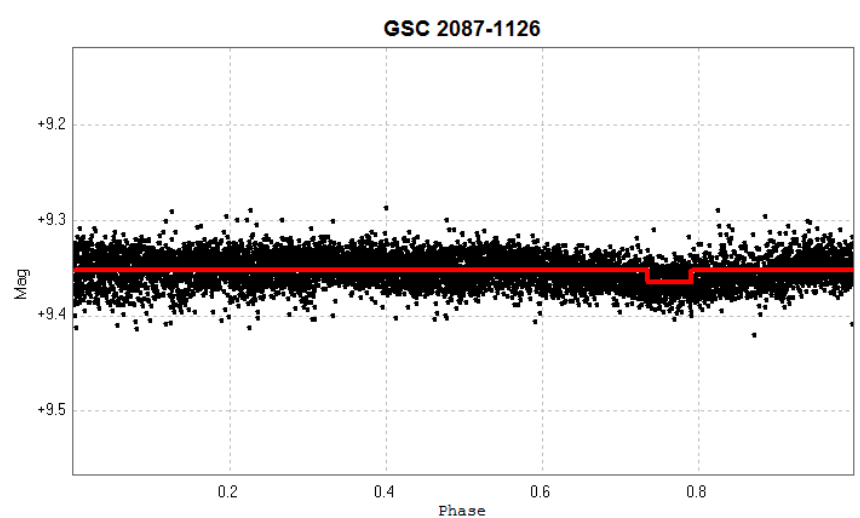

Figure 6. PERANSO phase diagram for GSC 2087-1126 produced by the BLS algorithm with a period of 0.79380 day.

Once we identify a light curve to have a possible transit, VARTOOLS (Hartman and Bakos, 2016) is used to run an EEBLS on the candidate light curve. VARTOOLS light curve analysis program is a free resource available for download that was developed by Joel Hartman of Princeton for manipulating large batches of light curves; the Windows-executable version was created in-house by one of our team members (A. Sweeney). The BLS algorithm outputs an assortment of useful information about the light curve found for a transit. A specified number of transit periods are calculated and listed in order of probability. The analysis yields the orbital period $P$ for GSC 2087-1126 b to be 0.7933028(7) day, where (7) represents the uncertainty in the last digit. The result is comparable to the period found using PERANSO software of 0.79330 day. The additional parameters from the model (synthetic) light curve produced by the algorithm are the transit duration after ingress $\left(Q_{\text {tran }}\right)$ as a fraction of the period, the ingress duration $\left(Q_{\text {ingress }}\right)$ as a fraction of the transit duration, and the depth of the transit in millimags $(\sqrt{\Delta F})$. Twice the ingress duration added to the transit duration yields the total transit duration $\left(t_{T}\right)$.

We use the Mandel-Agol algorithm (Mandel and Agol, 2002) in VARTOOLS to fit a model curve with limb-darkening to the phase curve.

Limb-darkening refers to the phenomenon where a star appears brighter in the center than around its edges. At the center of the stellar disk, the deepest and warmest layers which emit the most light are observed. At the limb, only the upper, cooler layers that produce less light can be seen. Therefore, since the outer edges of the star are less bright, less light from the star will be blocked by a planetary disk that is in front of the rim of stellar disk than by a planet that is in front of the center of the stellar disk. This phenomenon leads to a light curve that is more of a gradual curve in the ingress and egress phases, as one can see in Fig.7. This model fits the data better than the standard BLS model, since the data shows this gradual curvature as well. The transit parameters for calculation of the physical parameters, given in Table 2 are taken from the VARTOOLS Mandel-Agol model of GSC 2087-1126 b. The modeling analysis also indicates that 12 transits were observed over the campaign. We specifically observe the same area of the sky night after night to increase the chance of observing multiple transits and, therefore, to increase the signal-to-noise ratio.

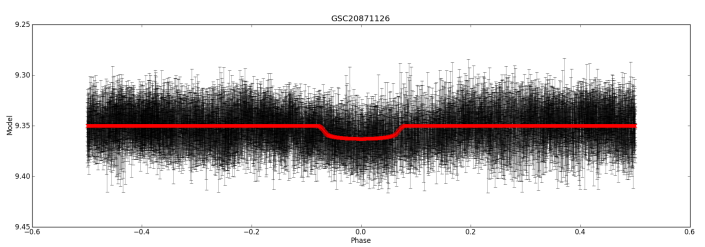

Figure 7. Phase plot for GSC 2087-1126 b with the Mandel-Algol model curve shown in red. The vertical axis gives the relative magnitude to the average in the $\mathrm{R}$ band.

The five physical parameters-the radius of the star $R_{*}$, the mass of the star $M_{*}$, the inclination of the 
orbit $i$, the semimajor axis of the orbit $a$, and the radius of the planet $R_{P}$-can be derived from the four measured parameters of the transit, $P, t_{F}, t_{T}$ and $\Delta F$ along with the stellar mass-radius relation $\rho / \rho_{\text {Sun }}=\left(\rho_{*} / \rho_{\text {Sun }}\right)^{1 /(1-3 x)}$. For Main Sequence stars $x \approx 0.8$. The relationships for the five physical parameters (Seager and Mallen-Ornelas, 2003) and the measured transit characteristics are shown in Fig.8.

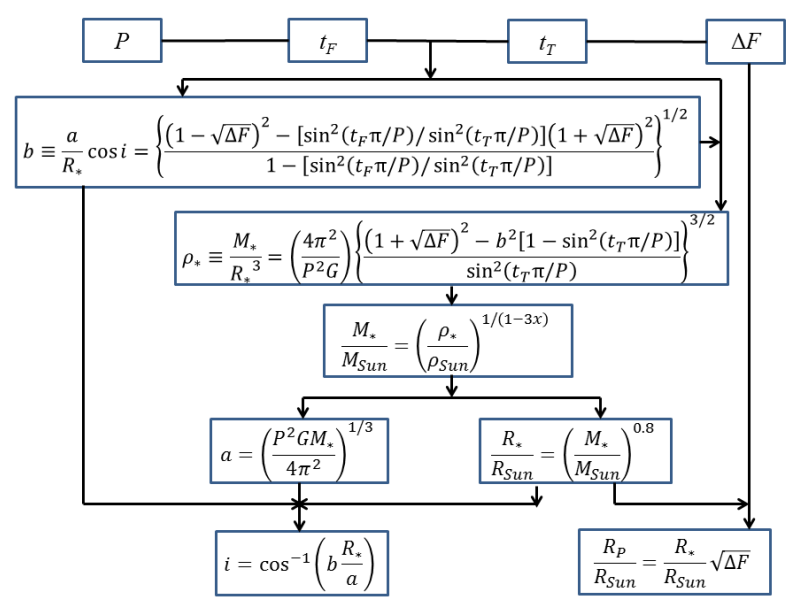

Figure 8. The five physical parameters-the radius of the star $R_{*}$, the mass of the star $M_{*}$, the inclination of the orbit $i$, the semimajor axis of the orbit $a$, and the radius of the planet $R_{P}-$ can be determined from four measured characteristics of the model transit light curve. For Main Sequence stars $x$ has the value of 0.8

Students also write a Python script to implement the exoplanet diagnostic of Tingley (Tingley and Sackett, 2005), which calculates the probability that a transit is due to a planet and not to a binary. We usually find main sequence spectral types $F$ through late $\mathrm{K}$, whose radii can vary by a factor of three or so, while the typical planetary radius that can produce a transit observable by ground-based search varies by about a factor of two. The Tingley diagnostic $\eta_{p}$ takes the radius of the planet to be constant and is given by

$\eta_{p}=\frac{D_{\text {obs }}}{2 Z(1+\sqrt{1.3 / d})}\left(\frac{2 \pi G M_{\text {Sun }}}{P}\right)^{1 / 3} R_{p}^{-7 / 12} R_{\text {Sun }}^{-5 / 12}\left(\frac{1.3}{d}\right)^{5 / 24}$

where $D_{o b s}$ is the measured duration of the transit, $d$ is the fractional depth of the transit, and $P$ is the orbital period. For an exoplanet event, $\eta_{p} \leq 1$ whereas $\eta_{p} \geq 1$ usually designates blending of stars, grazing binaries, or similar events.

\section{Results and Discussion}

Using the output of VARTOOLS BLS, students wrote a Python script to calculate the physical parameters of the star-planet system from the equations shown in Fig.8. As summarized in Tables 2 and 3, GSC 2087-1126 is very similar to the Sun, having a temperature of $5867 \mathrm{~K}$, a mass slightly greater than the Sun, at $1.48 \mathrm{M}_{\text {Sun }}$ and a radius 1.37 times that of the Sun. The average density of $1318 \mathrm{~kg} / \mathrm{m}^{3}$ is also similar to that of the Sun. In the USNO-B1.0 catalog Zacharias et al. (2013) reports GSC 2087-1126 as a spectral type FO main sequence star; the calculated mass, radius, and average density are less than that of a F0 main sequence star. However, since the calculated physical parameters are sensitive to the measured parameters, it is not unexpected that the values would not be exact. Table 2 also indicates that 12 transits were observed and the average transit depth was 12 mmag and the total transit duration of 0.15261 day with a period of 0.793300 (3) day.

Table 2. Transit parameters measured from modeling and stellar characteristics of GSC 2087-1126.

\begin{tabular}{ll}
\hline Measured & Value \\
\hline Period $P[\mathrm{~d}]$ & $0.793300(3)$ \\
$Q_{\text {tran }}$ & $0.14713(5)$ \\
$Q_{\text {in }}$ & $0.15375(5)$ \\
$\Delta F[\mathrm{mag}]$ & $0.01223(3)$ \\
$N_{\text {tran }}$ & 12 \\
$B$ & 12.34 \\
$V$ & 11.74 \\
Temp [K] & 5867 \\
Epoch [MJD] & $56071.84204(4)$ \\
\hline
\end{tabular}

Table 2 shows that the planet is 1.5 Jupiter diameters and orbits very close to a star at 0.019 $\mathrm{AU}$ which is approximately $1 / 20^{\text {th }}$ of the orbital radius of Mercury about the Sun. The planet is a hot Jupiter that orbits within the corona of the star. 
The angle of inclination of the system falls in the range $80^{\circ}-90^{\circ}$, which is what one would expect from a detectable transit. The Tingley diagnostic is 0.76 , which indicates the candidate is more likely a planetary transit than a binary and that it is worthy of subsequent follow-up studies for verification. In order to conduct follow-up studies, we need to know when a transit would occur in the future. The ephemeris of a transit refers to a time in the future when the object is predicted to transit again and it is calculated by taking the epoch of the transit-the Julian date of the first transit-and adding to it the period multiplied by the number of cycles desired, that is,

$J D=E_{0}+P \times E$,

We find the ephemeris for the transit midpoint for GSC 2087-1126 b to be given by

$$
J D=2456071.84204(4)+0.793300(3) \times E
$$

where $\mathrm{E}$ is the cycle number and $\mathrm{P}$ is the period in days. This ephemeris allows for subsequent follow-up on the candidate using larger telescopes that can observe single transits.

Table 3. Summary of characteristics of GSC 2087-1126 and the exoplanet candidate GSC 2087-1126 b.

\begin{tabular}{ll}
\hline Parameter & Value \\
\hline$b$ & $0.594(6)$ \\
$\rho\left[\mathrm{kg} / \mathrm{m}^{3}\right]$ & $1310(8)$ \\
$M * / M_{\text {Sun }}$ & $1.47(1)$ \\
$a[\mathrm{AU}]$ & $0.019(2)$ \\
$R * / R_{\text {Sun }}$ & $1.368(3)$ \\
inc $\left(^{0}\right)$ & $82.16(6)$ \\
$R_{p} / R_{J}$ & $1.50(1)$ \\
$\eta_{p}$ & 0.76 \\
\hline
\end{tabular}

\section{Summary}

The core experimental tactic of taking approximately 30 nights of data on the same starfield improves the signal-to-noise ratio by recording multiple transits and is shown to be a viable, successful approach to transit searches conducted with small aperture telescopes. Ground-based transits are finding hot Jupiters in 1 out of 30,000-50,000 stars. Our results are consistent with this statistic. Furthermore, realistic, physical results for the new transit candidate GSC 2087-1126 b have been obtained. Transits are not readily visible in a single night's light curve but rather only for period-folded light curves of data taken over multiple nights. The identification and investigation of the new exoplanet candidate gives an ephemeris and magnitude changes for follow-up studies with a larger telescope that can observe a single transit. Such follow-up observations-either photometrically or spectroscopically-are needed to confirm GSC 2087-1126 b as a new exoplanet.

\section{Acknowledgements}

The support of the Donald A. Cowan Institute and the Joe Oscar Neuhoff Fund at the University of Dallas is gratefully acknowledged. Access to the Monroe Observatory through the University of North Texas is greatly appreciated. Use of the Deep Sky Observatory Collaborative has made this work possible.

\section{Software}

Python, Numpy, The SkyX, Maxim DL, PinPoint, SExtractor, AIP4Win, PERANSO, VARTOOLS

\section{References}

Aumen, L., Olenick, R., and Sweeney, A. (2015). Discovery and Follow-up Observations of the Exoplanet Candidate GSC02087-01126. Bulletin of the American Physical Society, 60.

Berry, R. and Burnell, J. (2000). Astronomical Image Processing. Willman-Bell, Inc.

Bertin, E. and Arnouts, S. (1996). SExtractor: Software for source extraction. Astronomy and Astrophysics Supplement Series, 117(2):393-404. 
Cameron, A. C., Bouchy, F., Hébrard, G., Maxted, P., Pollacco, D., Pont, F., Skillen, I., Smalley, B., Street, R., West, R. G., et al. (2007). WASP-1b and WASP-2b: two new transiting exoplanets detected with SuperWASP and SOPHIE. Monthly Notices of the Royal Astronomical Society, 375(3):951-957.

Charbonneau, D., Brown, T. M., Latham, D. W., and Mayor, M. (1999). Detection of planetary transits across a sun-like star. The Astrophysical Journal Letters, 529(1):L45.

Denny, R. (2006). PINPOINT Astrometric Engine. DC-3 Dreams, SP, Mesa, AZ.

Hartman, J. D. and Bakos, G. Á. (2016). VARTOOLS: A program for analyzing astronomical time-series data. Astronomy and Computing, $17: 1-72$.

Haswell, C. A. (2010). Transiting exoplanets. Cambridge University Press.

Heuser, M. (2013). Evaluation of the EEBLS Algorithm in STExTS Exoplanet Searches. Thesis, University of Dallas.

Horne, J. H. and Baliunas, S. L. (1986). A prescription for period analysis of unevenly sampled time series. The Astrophysical Journal, 302:757-763.

Lomb, N. R. (1976). Least-squares frequency analysis of unequally spaced data. Astrophysics and Space Science, 39(2):447-462.

Mandel, K. and Agol, E. (2002). Analytic light curves for planetary transit searches. The Astrophysical Journal Letters, 580(2):L171.

Meier, J., Olenick, R., Sweeney, A., Schneiderjan, J., and Heuser, M. (2013). O-C Calculations for Two New Binaries and V1097 Herculis. Bulletin of the American Physical Society, 58(3).

Melendez, M., Olenick, R., and Sweeney, A. (2014). Blazhko Effect in a Newly Discovered and a Known RR Lyrae Star. Bulletin of the American Physical Society, 59(2).
Pepper, J., Pogge, R. W., DePoy, D., Marshall, J., Stanek, K., Stutz, A. M., Poindexter, S., Siverd, R., O'Brien, T. P., Trueblood, M., et al. (2007). The Kilodegree Extremely Little Telescope (KELT): A Small Robotic Telescope for Large-Area Synoptic Surveys. Publications of the Astronomical Society of the Pacific, 119(858):923.

Pont, F., Zucker, S., and Queloz, D. (2006). The effect of red noise on planetary transit detection. Monthly Notices of the Royal Astronomical Society, 373(1):231-242.

Scargle, J. D. (1982). Studies in astronomical time series analysis. II-Statistical aspects of spectral analysis of unevenly spaced data. The Astrophysical Journal, 263:835-853.

Schneiderjan, J., Olenick, R., Sweeney, A., Meier, J., and Heuser, M. (2013). PHOEBE Modeling of Three New Binaries in Hercules. Bulletin of the American Physical Society, 58(3).

Seager, S. and Mallen-Ornelas, G. (2003). A unique solution of planet and star parameters from an extrasolar planet transit light curve. The Astrophysical Journal, 585(2): 1038.

Tamuz, O., Mazeh, T., and Zucker, S. (2005). Correcting systematic effects in a large set of photometric light curves. Monthly Notices of the Royal Astronomical Society, 356(4):1466-1470.

Tingley, B. and Sackett, P. D. (2005). A photometric diagnostic to aid in the identification of transiting extrasolar planets. The Astrophysical Journal, 627(2):1011.

Vanmunster, T. (2007). PERANSO period analysis software.

Zacharias, N., Finch, C., Girard, T., Henden, A., Bartlett, J., Monet, D., and Zacharias, M. (2013). The fourth US naval observatory CCD astrograph catalog (UCAC4). The Astronomical Journal, 145(2):44. 